\title{
Introduction to the Special Issue on HRI Perspectives and Projects from around the Globe
}

\author{
Michael A. Goodrich, \\ Brigham Young University \\ and \\ Kerstin Severinson Eklundh \\ KTH Royal Institute of Technology
}

Human-robot interaction (HRI) is of global importance, as exemplified by the human and financial resources dedicated to research and development in the area. The vision for this special issue was to provide a broad, global perspective on the field. More specifically, the vision was for researchers in representative projects from around the world to share lessons and perspectives on HRI obtained from those projects.

We received a very positive response to the call for papers for this special issue. Submissions were received from Asia, Europe, Australia, North America, and South America. Many submissions passed a multiple-stage review process and received lengthy and detailed reviews from senior experts in the field.

We have grouped the contributions into three sets. Each article in the first set describes a large system that required the integration of many different types of technologies. The first paper in the first set is by D. Glas et al., and discusses a multi-year effort from Japan to design social robots for use in public spaces. The authors present a framework for organizing multiple social robots, and then use this framework to inform the design and control of multiple robots.

The second paper is by T. Belpaeme et al., and presents the ALIZ-E project, which is a broad European research effort that aims to achieve real-time interaction "in the wild" between a robot and a child user in a hospital setting. The objective is to develop and study long-term adaptive interaction between children and a robot companion. An evaluation scheme is presented that includes specific games and activities that support the robot's roles.

The third paper in this set is by J. Ruiz-del-Solar, and presents work from Chile on the design of a general-purpose robot that they call "Bender." Bender was designed to be used in schools, fairs, and similar environments, so the authors present an evaluation of what they state as a key element of its design, namely whether or not the robot could accurately express emotion.

The fourth paper is by E. Torta et al., and presents work from The Netherlands and Austria that aims to understand the attitudes of people toward socially assistive

\footnotetext{
Authors retain copyright and grant the Journal of Human-Robot Interaction right of first publication with the work simultaneously licensed under a Creative Commons Attribution License that allows others to share the work with an acknowledgement of the work's authorship and initial publication in this journal.
}

Journal of Human-Robot Interaction, Vol. 1, No. 2, 2012, Pages 1-2. DOI 10.5898/JHRI.1.2.Goodrich 
robots, particularly the growing number of elderly people who need assistance in their home. Starting with an exploration of user needs, the authors first report a sequence of WoZ-studies, and subsequently present field studies in Europe and Israel in a fully functional prototype of an intelligent home. The studies suggest that a socially assistive robot with a gesturing capability has a motivating effect on users in certain tasks, but that a conventional touch-screen interface is preferred for acquiring information.

The fifth paper in this first set is by XiaoPing Chen et al., and presents work from China on the construction of the OK-KeJia robot prototype. This prototype focuses on the extraction and utilization of knowledge in semi-structured and unstructured natural languages. The paper includes an evaluation of a portion of a large system designed to extract such knowledge.

The second set of articles consists of smaller systems than those presented in the first set, but ones that argue for a different paradigm or perspective on HRI. The first paper in this set is from Ferland et al., and presents feasibility studies from Canada of three innovative interaction techniques implemented on the humanoid robot IRL-1. The work involves a design paradigm of natural interaction, taking advantage of people's experience of direct physical interaction with the real world. One of the techniques tested with users focuses on the capability of the robot to be actively steered by pushing and pulling, a method that is compared with remote control.

The second paper in this set is by Kirchner and Alempijevic, and presents work from Australia that discusses a paradigm for human-robot interaction based on sociocontextual cues. The paradigm asserts that peer interaction necessitates a capability to read and perceive relevant aspects of the user and the environment. It also necessitates a capability to elicit cues designed to affect the behavior of the human user. The paper presents these components as parts of an interaction model or paradigm for HRI based on insights in the behavioral sciences.

The third paper in this set is by Harutyunyan et al., and presents work from Abu Dhabi in the United Arab Emirates. The paper introduces the notion of cognitive telepresence as a new design principle in HRI, with applications across various semiautonomous robots. The authors give various examples of the concept and show its relationships with other concepts such as situation awareness and telepresence. Furthermore, they discuss how to measure cognitive telepresence and suggest formal metrics that are used in example scenarios and a user study

Interestingly, all but one of the accepted contributions addressed a humanoid or social robot. The third set of articles, consisting of a single article, provides an important broadening to the special issue by providing a perspective on supervisory control of robots. This article, by Miller, puts forth a measurement perspective distilled from a North Atlantic Treaty Organization exercise. Although the article is interesting in its own right, the article is an important contribution to the special issue because supervisory control of air and ground robots is a far-reaching application of HRI from multiple countries.

Taken together, these papers demonstrate some of the global trends that have become evident in human-robot interaction in the past few years. These articles draw from a wide range of disciplines (including psychology, education, human factors, and linguistics) and make use of methodologies that are equally broad (including user studies, 
Wizard of Oz studies, field evaluations, and engineering evaluations). Similarly, there is an extremely wide range of robots employed in these studies - custom-built robots to offthe-shelf commercial systems, humanoid robots to non-anthropomorphic robots, autonomous control systems to remotely operated systems. This diversity of both platforms and disciplinary connections reflects the youth and diversity of the current HRI research environment.

We selected an unusually high number of contributions, nine in all, but we felt that each paper was needed to provide a global perspective. Even so, the perspective is admittedly incomplete since the world is, after all, a very big place. Notably missing are a broader set of papers addressing non-social robotic systems.

Many thanks go to the generous manuscript submitters, reviewers, and editors, who made this special issue possible. We also appreciate the financial assistance or inkind support given by various contributors to make the journal free for readers and authors; this support allows this treatment of the global perspective to reach a global audience. We hope that these articles will be as insightful and useful for you as they are for us.

Guest editors' contact information: Michael A. Goodrich, Computer Science Department, Brigham Young University, Provo, Utah, USA. Email: mike@cs.byu.edu. Kerstin Severinson Eklundh, School of Computer Science and Communication, KTH Royal Institute of Technology, Stockholm, Sweden. Email: kse@csc.kth.se 